\title{
MONTE CARLO SIMULATION OF NEUTRONICS START-UP TESTS AT CHINA EXPERIMENTAL FAST REACTOR (CEFR)
}

\author{
Jiwon Choe1,2, Chirayu Batra1, Vladimir Kriventsev1, and Deokjung Lee2 \\ 1International Atomic Energy Agency, \\ Vienna International Centre, PO Box 100, 1400 Vienna, Austria \\ 2Department of Nuclear Engineering, Ulsan National Institute of Science and Technology \\ 50 UNIST-gil, Ulsan, 44919, Republic of Korea \\ chi91023@unist.ac.kr, C.Batra@iaea.org, V.Kriventsev@iaea.org, deokjung@unist.ac.kr
}

\begin{abstract}
China Experimental Fast Reactor (CEFR) is a small size sodium-cooled fast reactor (SFR) with a high neutron leakage core fueled by uranium oxide. The CEFR core with $20 \mathrm{MW}(\mathrm{e})$ power reached its first criticality in July 2010 , and several start-up tests were conducted from 2010 to 2011. The China Institute of Atomic Energy (CIAE) proposed to release some of the neutronics start-up test data for the IAEA benchmark within the scope of the IAEA's coordinated research activities through the coordinated research project (CRP) on "Neutronics Benchmark of CEFR Start-Up Tests", launched in 2018. This benchmark aims to perform validation and verification of the physical models and the neutronics simulation codes by comparing calculation results against collected experimental data.

The six physics start-up tests considered for this CRP include evaluation of the criticality, control rod worth, void reactivity, temperature coefficient, swap reactivity, and foil irradiation. Twenty-nine participating research organizations are performing independent blind calculations during the first phase of the project. As a part of this coordinated research, IAEA performed neutronics calculations using Monte Carlo code SERPENT.

Two kinds of 3D core models, homogenous and heterogeneous, were calculated using SERPENT, with ENDF/B-VII.0 continuous energy library. Preliminary results with a reasonably good estimation of criticality, as well as theoretically sound results of other five test cases, are available. The paper will discuss the core modelling assumptions, challenges and key findings of modelling a dense SFR core, preliminary results of the first phase of the $\mathrm{CRP}$, heterogeneity impact analysis between homogenous core models and heterogeneous core models and future work to be performed as a part of this four-year project.
\end{abstract}

KEYWORDS: Sodium Cooled Fast Reactor, CEFR, SERPENT, Monte-Carlo

\section{INTRODUCTION}

Many fast reactors (FRs) experiments have been carried out for several decades in various countries [2]; however, only a few neutronics benchmarks are available [3]. The CEFR core with $20 \mathrm{MW}(\mathrm{e})$ power reached its first criticality in July 2010 [4], and several start-up tests were conducted from 2010 to 2011. The China Institute of Atomic Energy (CIAE) proposed some of the neutronics start-up test data for the IAEA benchmark within the scope of the IAEA's coordinated research activity [1]. The coordinated research 
project (CRP) on "Neutronics Benchmark of CEFR Start-Up Tests" was launched in 2018. This benchmark aims to perform validation and verification of the physical models and the neutronics simulation codes by comparing calculation results against collected experimental data. Twenty-nine participating research organizations are performing independent blind calculations. The main objective of this benchmark is to improve understanding of the start-up of SFRs and validate the state-of-the-art fast reactor analysis computer codes against the recent experimental data obtained at the modern experimental SFR.

As a part of this coordinated research, IAEA performed neutronics calculations using a Monte Carlo (MC) codes, SERPENT [5], which can minimize modelling assumptions and produce reference solutions for code verification. Two 3D core models, heterogeneous and homogenous core, are modelled by SERPENT 2.1.27 with ENDF/B-VII.0 continuous-energy cross-section library [6]. Five out of six physics start-up tests considered for this paper include evaluation of the criticality, control rod worth, void reactivity, temperature coefficient, and swap reactivity. Preliminary results with a well-estimated criticality, as well as reasonably good estimation of the other four cases, are available. The paper will discuss the core modelling assumptions, challenges of modelling a dense SFR core, the impact of heterogeneity, preliminary results of the first phase of this CRP, and future work to be performed as a part of this four-year project. It is a crucial benchmark exercise for validation of physical and mathematical neutronics models implemented in SFR simulation codes, which will help in reducing uncertainty in modelling of neutronics in fast reactors.

\section{MODELLING CEFR BENCHMARK}

Table 1 summarizes the main parameters of the CEFR of the first loading at $20^{\circ} \mathrm{C}$ [7]. The CEFR is a small size reactor with $65 \mathrm{MW}$ thermal power. The designed life is 30 years, and the refuelling period is 80 days. 79 fuel subassemblies (SAs) with $\mathrm{UO}_{2}$ are loaded at the initial core. The overall core height is $850 \mathrm{~mm}$, which consists of a fuel region of $450 \mathrm{~mm}$ and $350 \mathrm{~mm}$ blanket region at above and below. Fuel and blanket use different fuels: annular fuel pellets using $\mathrm{UO}_{2}$ fuel with $64.4 \mathrm{w} / \mathrm{o}{ }_{235} \mathrm{U}$ and traditional fuel pellets using $\mathrm{UO}_{2}$ fuel with $0.3 \mathrm{w} / \mathrm{o} 235 \mathrm{U}$, respectively. Neutron absorber material is $\mathrm{B}_{4} \mathrm{C}$ with different ${ }_{10} \mathrm{~B}$ enrichment in boron shielding SAs and control rod SAs. The core layout of the operation loading is shown in Figure 1.

Table 1. Core Specification of CEFR

\begin{tabular}{|c|c|}
\hline Parameters & Value \\
\hline Thermal power / Electric power [MW] & $65 / 20$ \\
\hline Core height [mm] & 450 \\
\hline Equivalent core diameter [mm] & 600 \\
\hline Height of lower blanket [mm]/ upper blanket [mm] & $250 / 100$ \\
\hline SA lattice pitch [mm] & 61 \\
\hline Number of fuel SAs (1st Loading) & 79 \\
\hline 235 U enrichment of fuel / blanket [\%(wt)] & $64.4 / 0.3$ \\
\hline 10 B enrichment of Regulating SA / Shim \& Safety SA, Boron shielding SA & $92 /$ Natural \\
\hline
\end{tabular}

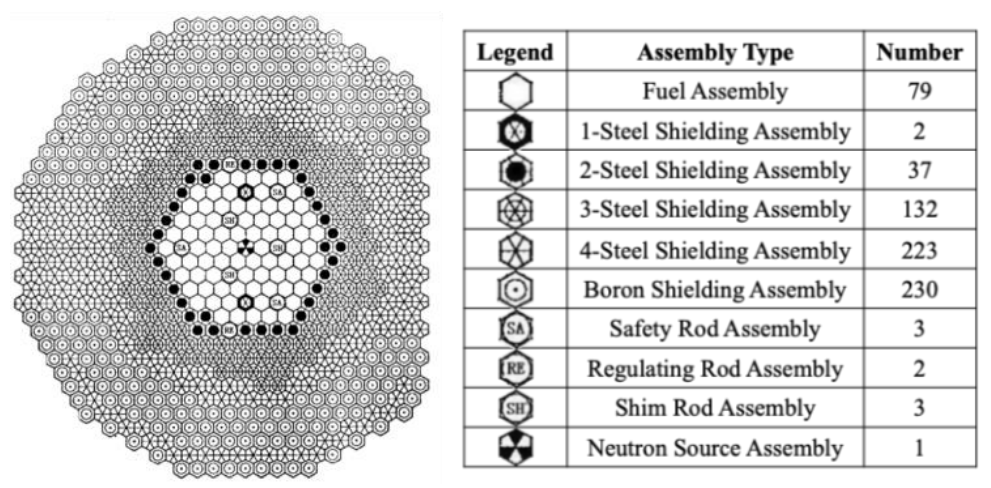

Figure 1. Core Configuration 
Thermal expansion is not considered for both heterogeneous and homogenous core model, and therefore, the geometry and solid material are referred at $20^{\circ} \mathrm{C}$, however, sodium density changes as a function of temperature. Fuel loading amount and $\mathrm{B} 4 \mathrm{C}$ amount are calculated by measurement mass. Axial height of the model is around $190 \mathrm{~cm}$ from the grid plate to upper shielding of SS SA, ignoring the rest of the assembly parts. The spacer wire is smeared into the cladding for heterogeneous core, with volume preservation. For the homogeneous core, the materials in assembly and duct out of sodium are homogenized by preserving their volume fractions. Figure 1 and 2 show representative homogenous and heterogeneous models made using SERPENT. The cross-sections used in the calculations were generated at $300 \mathrm{~K}$; however, various temperatures are interpolated by the Doppler-preprocessor. Obviously, the cell number of the homogenous core is much smaller than that of the heterogeneous core, therefore, homogenous calculations have been performed with a lower number of particles to save computational time. The eigenvalue calculations for heterogeneous and homogenous models are performed using 500 inactive and 2,000 active cycles with 200,000 source neutrons, and 500 inactive cycles and 2,000 active cycles with 50,000 source neutrons respectively. With these neutron histories, the standard deviations of eigenvalues are $6 \mathrm{pcm}$ for the heterogenous core and $10 \mathrm{pcm}$ for the homogeneous core, respectively. Moreover, all uncertainties of the following results are given at $1-\sigma$.

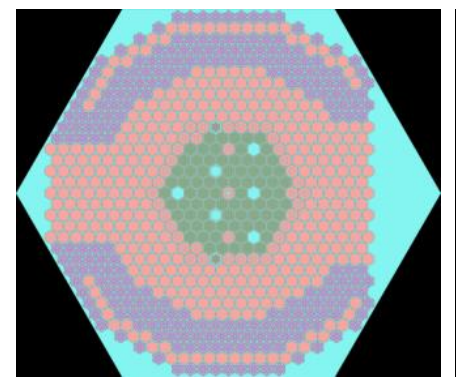

XY-plane

(a) Heterogenous

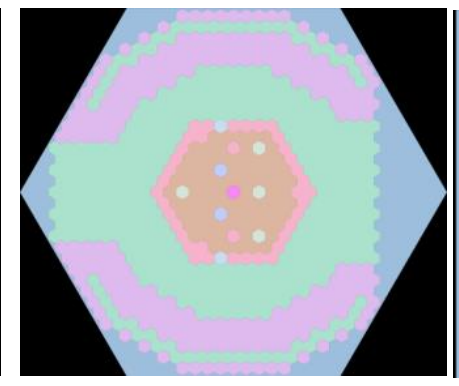

XY-plane

(b) Homogenous

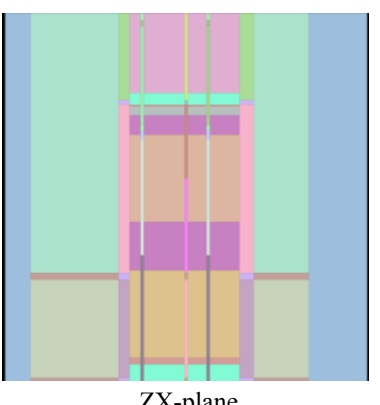

ZX-plane
ZX-plane

Figure 2. SERPENT-2 Cross-sectional View of CEFR heterogeneous (left) and homogenous (right)Core

\subsection{Criticality Test}

\section{BENCHMARK RESULTS}

The start-up was performed with 79 mock-up fuel SAs at the start, with all control rods out-of-core. The core remained sub-critical with 71 fuel SAs and turned super-critical with 72 nd assembly load. The regulating assembly (RE2) was used to keep the core critical state, and criticality was reached with RE2 position at $70 \mathrm{~mm}$ [7]. Critical test results are summarized in Table 2. At the predicted critical state, the reactivities of the heterogeneous core model and homogenous core model are $797 \mathrm{pcm}$ and $751 \mathrm{pcm}$, respectively. Both cases overestimate the criticality. The case of '71 fuel SA with ARO' shows a small heterogeneity effect of fuel SAs (90 pcm).

Table 2. Criticality test results

\begin{tabular}{|c|c|c|c|c|c|}
\hline \multirow{2}{*}{ \# of loaded FAs } & \multirow{2}{*}{$\begin{array}{c}\text { RE2 Rod position } \\
{[\mathrm{mm}]}\end{array}$} & \multirow{2}{*}{ Core state } & \multicolumn{3}{|c|}{ k-effective } \\
\cline { 4 - 6 } & & & Hetero \pm 0.00006 & Homo \pm 0.00010 & Diff. [pcm] \\
\hline 71 & Out-of-core & subcritical & 1.00570 & 1.00480 & -90 \\
\hline 72 & 190 & supercritical & 1.00832 & 1.00801 & -31 \\
\hline 72 & 170 & supercritical & 1.00835 & 1.00818 & -17 \\
\hline 72 & 151 & supercritical & 1.00817 & 1.00812 & -5 \\
\hline 72 & 70 & predicted critical & 1.00797 & 1.00751 & -46 \\
\hline
\end{tabular}


The flux spectrum of homogenous core model is slightly softer than that of heterogeneous core as shown in Figure 3, and is a probable reason for lower k-eff. Radial power distributions are the same within 1- $\sigma$.

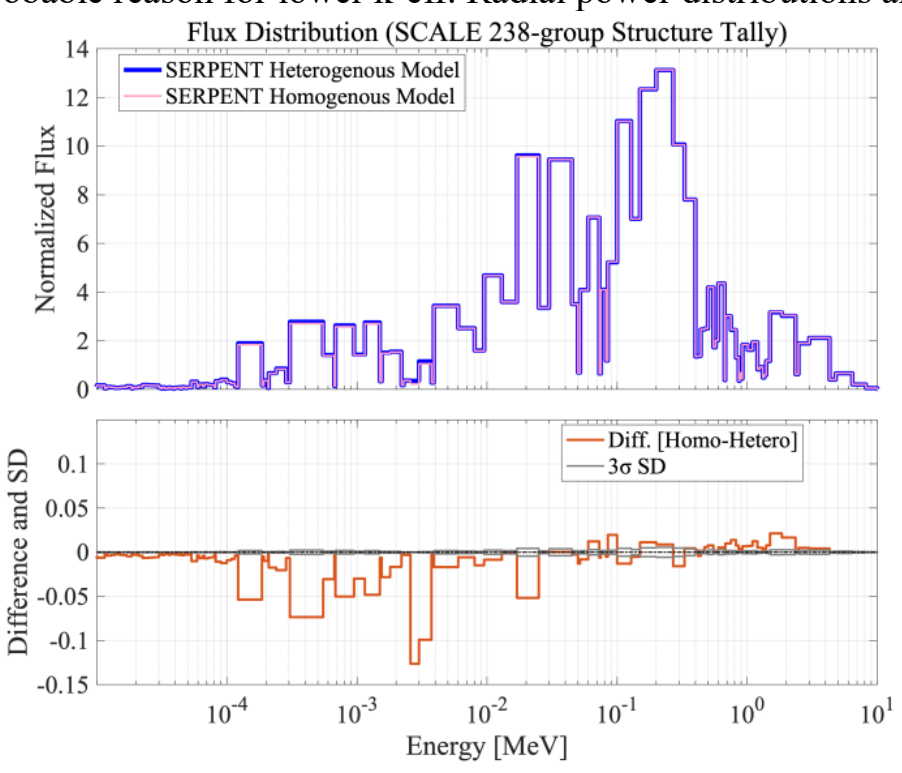

Figure 3. Normalized neutron flux distribution comparison as function of energy. (Predicted Critical State with 72 FAs, RE2 at $70 \mathrm{~mm}$ )

\subsection{Control Rod Worth}

The control rod worth measurement experiment was carried out at cold state $\left(250^{\circ} \mathrm{C}\right)$ in operation layout with 79 fuel SAs shown in Figure 1. The control assemblies have same dimensions and materials with a difference in $10 \mathrm{~B}$ enrichment in $\mathrm{B} 4 \mathrm{C}$ : $92 \mathrm{w} / \mathrm{o} 10 \mathrm{~B}$ for regulating $\mathrm{SAs}(\mathrm{RE})$ and natural ${ }_{10} \mathrm{~B}$ abundance for shim SAs (SH) and safety SAs (SA). Table 3 provides exact positions during each rod-drop experiment, referenced from the bottom of the fuel region [7].

The integral worth is defined as the total reactivity change when one or several control rods move from top to the bottom position. Regulating SAs with natural $10 \mathrm{~B}$ and located mostly at the outside of active core have the least worth among all three types of control SAs. The safety SAs are located near the reflector periphery, and location of the shim SAs is closer to the centre of the core. Therefore, the safety SAs worth is higher than the shim SAs, even with the same dimensions and materials. For the same type of control SAs, i.e., shim rods, rod worth is different due to the asymmetric core loading pattern. Overall, the homogenous core model overestimates rod worth by around $10 \%$ than that of the heterogeneous core model. In order to calculate reactivities, such as temperature coefficients, sodium void worth, and swap reactivity, differential rod worth is also calculated as shown in Figure 4. The differential rod worth of homogenous core is higher than that of the heterogeneous core; thus, the rod worth change can lead to overestimation of reactivity coefficient calculation. The differential rod worth is calculated as:

$$
\Delta \rho_{D}^{j}=\left(k_{j+1}-k_{j}\right) /\left(k_{j+1} k_{j} \Delta H\right) \times 10^{5}[\mathrm{pcm} / \mathrm{mm}], j=1 \ldots N-1
$$

where $\Delta \rho_{D}^{j}$ is differential rod worth at $j$ step, $\Delta H$ is the change of control rod position between $j$ and $j+1$ steps. The differential rod worth will be used for reactivity coefficients calculation. The reactivity coefficients, such as temperature reactivity, sodium void worth and swap reactivity, are calculated by combining reactivity of control rod change and reactivity change of criticality as follows:

$$
\Delta \rho_{\text {reactivity }}=\Delta \rho_{\text {criticality }}+\sum_{i=C R s} \Delta \rho_{D}^{i} \cdot \Delta H^{i}
$$


where $\Delta \rho_{\text {reactivity }}$ is the type of reactivity coefficients, $i$ is the type of control rods, $\Delta \rho_{D}^{i}$ is differential rod worth of control rod type $i, \Delta H^{i}$ is the change of rod position of control rod type $i$, and $\Delta \rho_{\text {criticality }}$ is the reactivity change of the criticality state.

Table 3. Control Rod Worth

\begin{tabular}{|c|c|c|c|c|c|c|c|c|c|c|c|c|}
\hline \multirow[b]{2}{*}{ Rod or rod group } & & \multicolumn{8}{|c|}{ Control rod positions from bottom of fuel region[mm] } & \multicolumn{3}{|c|}{ Rod Worth [pcm] } \\
\hline & & RE1 & RE2 & $\mathrm{SH} 1$ & $\mathrm{SH} 2$ & $\mathrm{SH} 3$ & SA1 & SA2 & SA3 & $\begin{array}{l}\text { Hetero } \\
\pm 10\end{array}$ & $\begin{array}{c}\text { Homo } \\
\pm 18\end{array}$ & $\begin{array}{c}\text { Diff. } \\
{[\mathrm{pcm}]}\end{array}$ \\
\hline \multirow{2}{*}{ RE1 } & $\mathrm{B}$ & 501 & 106 & 240 & 240 & 239 & 498 & 500 & 500 & \multirow{2}{*}{158} & \multirow{2}{*}{165} & \multirow{2}{*}{7} \\
\hline & $\mathrm{A}$ & -1 & 106 & 240 & 240 & 239 & 498 & 500 & 500 & & & \\
\hline \multirow{2}{*}{ RE2 } & $\mathrm{B}$ & 106 & 499 & 240 & 240 & 239 & 498 & 500 & 500 & \multirow{2}{*}{140} & \multirow{2}{*}{184} & \multirow{2}{*}{44} \\
\hline & $\mathrm{A}$ & 106 & 5 & 240 & 240 & 239 & 498 & 500 & 500 & & & \\
\hline \multirow{2}{*}{ SH1 } & B & 240 & 240 & 501 & 141 & 141 & 498 & 499 & 499 & \multirow{2}{*}{1890} & \multirow{2}{*}{2125} & \multirow{2}{*}{235} \\
\hline & $\mathrm{A}$ & 240 & 240 & 4 & 141 & 141 & 498 & 499 & 499 & & & \\
\hline \multirow{2}{*}{$\mathrm{SH} 2$} & $\mathrm{~B}$ & 239 & 240 & 151 & 498 & 151 & 498 & 500 & 500 & \multirow{2}{*}{1849} & \multirow{2}{*}{2067} & \multirow{2}{*}{217} \\
\hline & \begin{tabular}{|l|}
$\mathrm{A}$ \\
\end{tabular} & 239 & 240 & 151 & -1 & 151 & 498 & 500 & 500 & & & \\
\hline \multirow{2}{*}{ SH3 } & $\mathrm{B}$ & 240 & 239 & 148 & 150 & 498 & 498 & 500 & 500 & \multirow{2}{*}{1846} & \multirow{2}{*}{2073} & \multirow{2}{*}{227} \\
\hline & \begin{tabular}{|l|}
$\mathrm{A}$ \\
\end{tabular} & 240 & 239 & 148 & 150 & 7 & 498 & 500 & 500 & & & \\
\hline \multirow{2}{*}{ SA1 } & $\mathrm{B}$ & 240 & 239 & 240 & 240 & 241 & 498 & 499 & 499 & \multirow{2}{*}{916} & \multirow{2}{*}{1043} & \multirow{2}{*}{127} \\
\hline & $\mathrm{A}$ & 240 & 239 & 240 & 240 & 241 & 46 & 499 & 499 & & & \\
\hline \multirow{2}{*}{ SA2 } & B & 240 & 240 & 240 & 240 & 240 & 498 & 499 & 499 & & & \\
\hline & A & 240 & 239 & 240 & 240 & 240 & 498 & 55 & 499 & 891 & 1006 & 115 \\
\hline $\mathrm{SA3}$ & $\mathrm{B}$ & 240 & 239 & 240 & 240 & 240 & 498 & 499 & 499 & 065 & 1002 & 127 \\
\hline SA3 & $\mathrm{A}$ & 240 & 239 & 240 & 240 & 240 & 498 & 499 & 40 & 905 & 1092 & 121 \\
\hline $2 * \mathrm{CH}+2 * \mathrm{PF}$ & $\mathrm{B}$ & 247 & 247 & 239 & 240 & 239 & 498 & 500 & 499 & 3019 & 3400 & 381 \\
\hline $3^{*} \mathrm{SH}+2^{*} \mathrm{KE}$ & $\mathrm{A}$ & 0 & 5 & 1 & -1 & 7 & 498 & 500 & 499 & 3019 & 3400 & 381 \\
\hline & $\mathrm{B}$ & 247 & 248 & 501 & 141 & 141 & 498 & 500 & 499 & & & \\
\hline SH2 & $\mathrm{A}$ & -2 & 2 & 501 & -3 & 16 & 498 & 500 & 499 & 1003 & 1157 & 154 \\
\hline & $\mathrm{B}$ & 247 & 249 & 240 & 240 & 240 & 498 & 500 & 499 & & & \\
\hline $3 \nrightarrow S A$ & $\mathrm{~A}$ & 247 & 249 & 240 & 240 & 240 & 46 & 56 & 40 & 2956 & 3340 & 384 \\
\hline SA1+SA2 & B & 247 & 248 & 240 & 240 & 240 & 498 & 500 & 500 & 1903 & 2149 & \\
\hline SAI+SA2 & A & 247 & 248 & 240 & 240 & 240 & 45 & 54 & 500 & 1903 & 2149 & 240 \\
\hline & $\mathrm{B}$ & 247 & 248 & 240 & 240 & 240 & 499 & 500 & 500 & & & \\
\hline $2 * \mathrm{R}$ & A & 0 & 3 & 2 & -2 & 0 & 45 & 56 & 40 & 29 & 6558 & 729 \\
\hline 2 & $\mathrm{~B}$ & 248 & 248 & 500 & 141 & 141 & 498 & 500 & 499 & 4 & & \\
\hline & $\mathrm{A}$ & -2 & 2 & 500 & -3 & 7 & 45 & 55 & 40 & 4 & 4319 & 443 \\
\hline
\end{tabular}
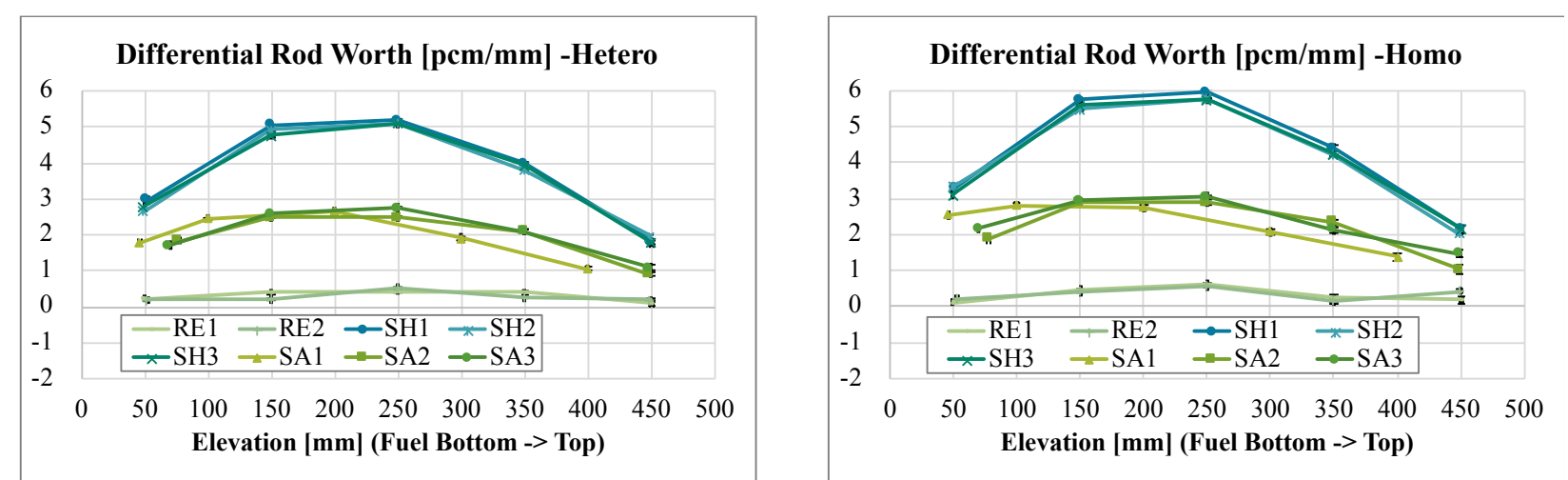

Figure 4. Differential Rod Worth Comparison 


\subsection{Temperature Coefficients}

The measurements were done at the basic core in operation loading with 79 FAs. Five sets of data were obtained by increasing the temperature; $250,275,283,293$, and $300^{\circ} \mathrm{C}$. The SA control rod positions are out-of-core, and the SH and RE control rod positions are moved to search criticality. Temperatures for solid materials are set as same as the sodium coolant temperature without change of density and dimension, but sodium coolant density changes according to the given temperature.

The temperature coefficient is calculated by a gradient of reactivity change along with temperature, as shown in Figure 5. Sodium density decreases with the increase in temperature, and this hardens the flux spectrum, leading to a negative temperature coefficient.

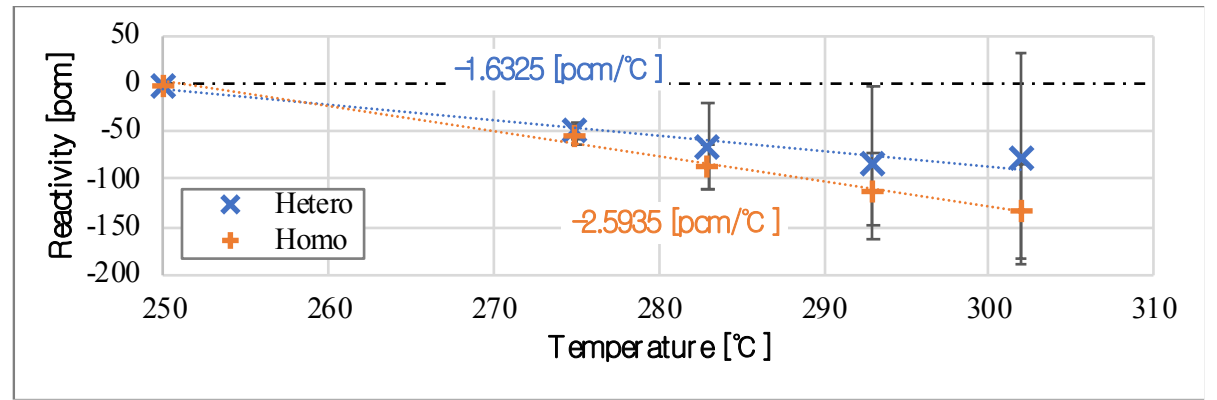

Figure 5. Temperature Coefficient Calculation Comparison

\subsection{Void Worth}

Sodium void reactivity is measured by replacing a fuel SA by vacuum-sealed SA and searching for the critical position of control rods. Five different fuel SA locations, as shown in Figure 6, were measured. Axial positions of control rods, SH and SA, are fixed at the middle of the core, with RE rods moving to attain criticality. Void worth results are shown in Figure 8. Sodium void reactivity at all regions is negative due to neutron. All values for five positions are similar due to two reasons: 1) the centre of the core has more neutrons and less leakage, 2) the outside of the core has more leakage. Heterogeneous core model underestimated the neutron leakage of voided assembly; however, it is difficult to evaluate it due to the large standard deviation.

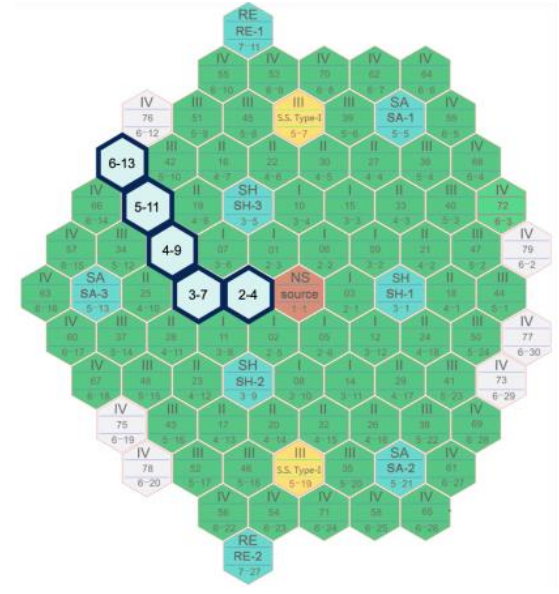

Figure 6. Positions and Void FA Loading for Void Reactivity Measurement.

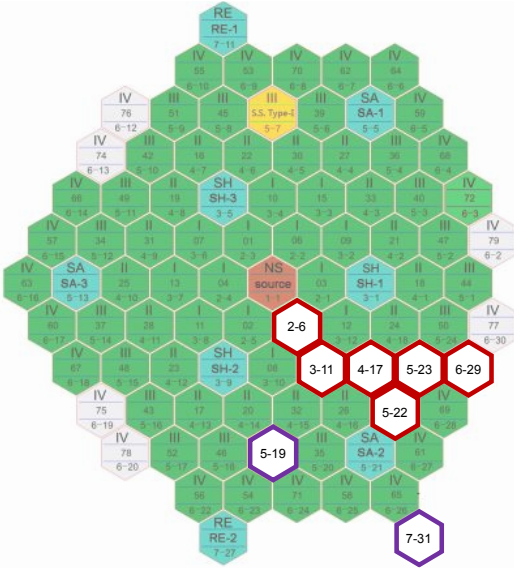

Figure 7. Positions and SA Loading for Swap Reactivity Measurement.

(Red: fuel SAs, Purple: Type-I SS SA) 


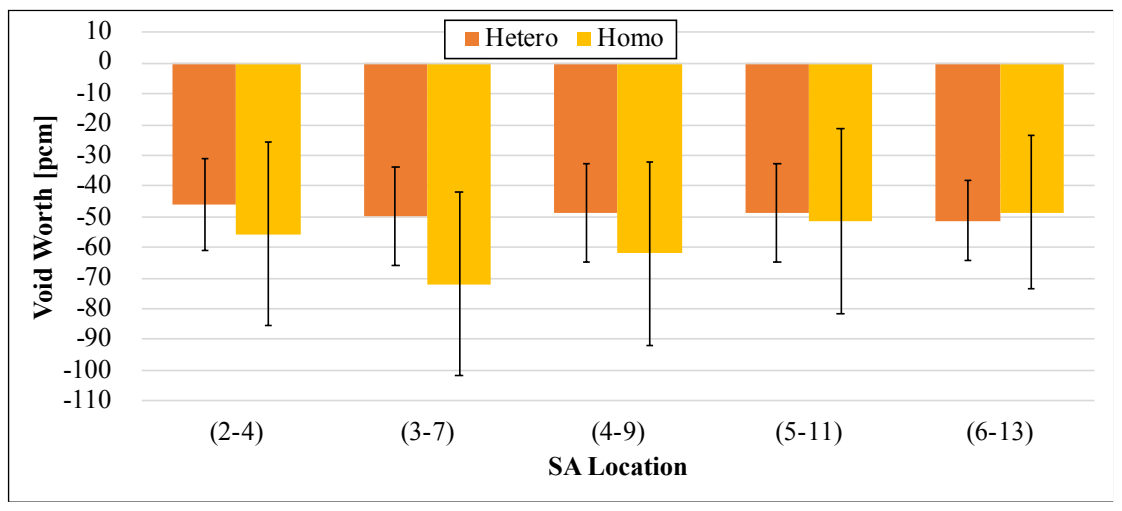

Figure 8. Void Worth Results Comparison

\subsection{Swap Reactivity}

Eight positions are selected for the measurements: 6 fuel SAs and 2 Type-I SS SAs, as shown in Figure 7. Fuel SA is replaced by SS SA, and SS SA is switched with one fuel SA. Other SAs are not changed when an SS SA is loaded at fuel SA position. Meanwhile, one SS SA is loaded instead of fuel SA for a safe measurement when a fuel SA is loaded at SS SA position. The SA control rods are at out-of-core, and the $\mathrm{RE}$ and $\mathrm{SH}$ control rods move to attain criticality.

Swap reactivities are measured in two ways, with more than two control rods moving to find the criticality of the core in 'Multiple Rods' case, and only one control rod moving in the 'Single Rod' case. Swap reactivities of fuel SA always show more negative reactivity (around $-300--800 \mathrm{pcm}$ ) due to loss of fuel, whereas swap reactivities of SS SA are closer to zero. Also, the closer the swapping is to the centre of the core, the more is the negative reactivity, as loss of fuel in the centre region needs more compensation. Measurement of SS SA swap reactivity is switching positions of fuel SA and SS SA; thus, fuel SA moves to the outside of the core, while SS SA moves inside. In both (7-31) and (5-19) cases, swap reactivities are small but still minus. In the other six cases, the absolute value of fuel SA swap reactivity increases near the centre. Therefore, loading fuel SA outside the core can cause lower excessive reactivity even though the same number of fuel SA is used in the reactor core. Both 'Multiple Rods' and 'Single Rod' results show the same trend; with the heterogeneous core model slightly overestimating the swap reactivities.

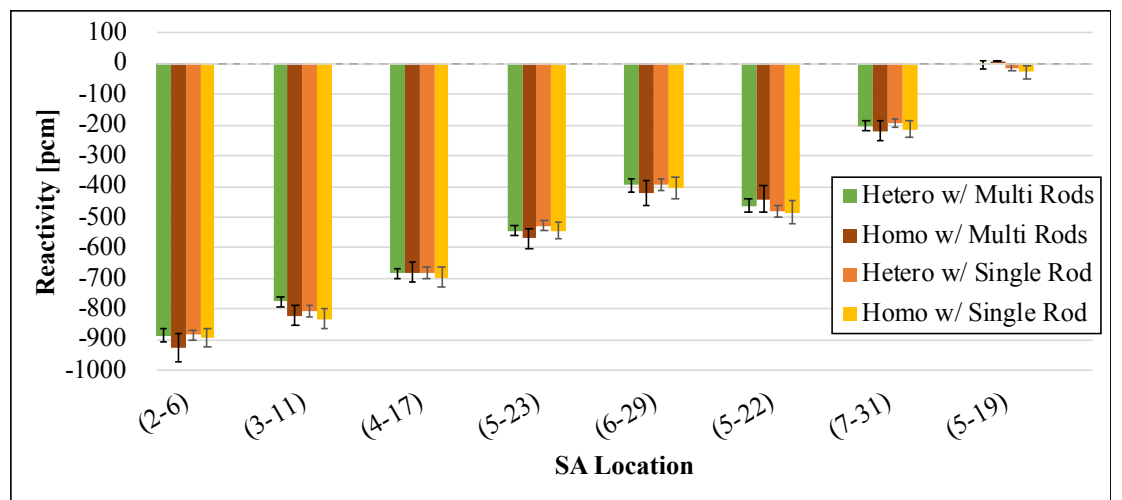

Figure 7. Swap Reactivity Results Comparison. 


\section{CONCLUSIONS}

As a part of the IAEA's Coordinated Research Project (CRP) the "neutronics benchmark of CEFR start-up tests," IAEA performed neutronics calculations using Monte Carlo codes SERPENT with the ENDF/BVII.0 cross-section library. Purpose of modelling the heterogeneous and homogenous core is to see the impact of heterogeneity of CEFR. Five of the six start-up tests within the scope of the benchmark are simulated: evaluation of the criticality, control rod worth, void reactivity, temperature coefficient, and swap reactivity. Especially, reactivity or coefficients is calculated with the control rod worth and criticality in the same manner as the measurement procedure.

From the evaluation of the core criticality using 72 fuel SAs, both the heterogeneous core and the homogenous core overestimates criticality by approximately $791 \mathrm{pcm}$ and $750 \mathrm{pcm}$. It has been found with some more calculations that the change of the cross-section library can reduce these high estimations: using the ENDF/VII.1 library lessens $400 \mathrm{pcm}$ of reactivity due to the cross-section change of some isotopes in structure materials. The k-eff of heterogeneous core model is higher by around $50 \mathrm{pcm}$ than that of homogenous core model due to harder flux spectrum. Nevertheless, the heterogeneity effect of fuel SAs is small. Integral rod worth and differential rod worth are calculated for safety evaluation and coefficient calculation, respectively. The control rod worth of the heterogeneous core is always lower than that of homogenous core. The heterogeneity impact of control rod worth is higher than that of fuel SAs. Void reactivity is negative wherever a vacuum SA instead of sodium replaces a specific fuel SA. The temperature coefficient also shows the negative value at the cold state $\left(250-302{ }^{\circ} \mathrm{C}\right)$, and it means that negative reactivity incomes as an increment of the reactor core temperature. Swap reactivity can estimate excessive reactivity change due to incorrect SAs loading. All the results have a good agreement, and the homogenous core always overestimated reactivities. For future work, all the cases will be re-calculated with more neutron histories to reduce statistical error. Furthermore, thermal expansion will be considered in order to be evaluated with experimental data.

\section{REFERENCES}

1. IAEA, D. of N. E. (2018). IAEA CRP: Neutronics Benchmark of CEFR Start-Up Tests (I31032). Retrieved from https://www.iaea.org/newscentre/news/new-crp-neutronics-benchmark-of-cefr-startup-tests-i31032

2. IAEA (2013). Status of Fast Reactor Research and Technology Development, IAEA-TECDOC-1691, IAEA, Vienna. Retrieved from https://www.iaea.org/publications/8667/status-of-fast-reactor-researchand-technology-development

3. Stauff, N. E., Kim, T. K., Taiwo, T. A., Buiron, L., Rimpault, G., Brun, E., Gulliford, J. (2016). Benchmark for Neutronic Analysis of Sodium-cooled Fast Reactor Cores with Various Fuel Types and Core Sizes.

4. IAEA. (2019). Power Reactor Information System: CEFR. Retrieved from https://pris.iaea.org/PRIS/CountryStatistics/ReactorDetails.aspx?current=1047

5. Leppänen, J., Pusa, M., Viitanen, T., Valtavirta, V., \& Kaltiaisenaho, T. (2015). The Serpent Monte Carlo code: Status, development and applications in 2013. Annals of Nuclear Energy, 82, 142-150.

6. Chadwick, M. B., Obložinský, P., Herman, M., Greene, N. M., McKnight, R. D., Smith, D. L., ... van der Marck, S. C. (2006). ENDF/B-VII.0: Next Generation Evaluated Nuclear Data Library for Nuclear Science and Technology. Nuclear Data Sheets, 107(12), 2931-3060.

7. Huo, Xingkai;Hu, Y., Chen, X., Xu, L., ... Duan, T. (2018). Technical Specifications for Neutronics Benchmark of CEFR Start-up Tests (CRP-I31032) V6.0. Beijing, China.

8. Forget, B., Xu, S., \& Smith, K. (2014). Direct Doppler broadening in Monte Carlo simulations using the multipole representation. Annals of Nuclear Energy, 64, 78-85.

9. Viitanen, T. (2009). Implementing a Doppler-preprocessor of cross section libraries in reactor physics code Serpent. M. Sc. Thesis, Helsinki University of Technology.

10. Yang, W. S. (2012). FAST REACTOR PHYSICS AND COMPUTATIONAL METHODS. Nuclear Engineering and Technology, 44(2), 177-198. 